\title{
Simulating pedigrees ascertained for multiple disease-affected relatives
}

\author{
Christina Nieuwoudt ${ }^{1}$, Samantha J. Jones ${ }^{2,3}$, Angela Brooks-Wilson ${ }^{2,4}$ and Jinko Graham ${ }^{1 *}$
}

\begin{abstract}
Background: Studies that ascertain families containing multiple relatives affected by disease can be useful for identification of causal, rare variants from next-generation sequencing data.

Results: We present the R package SimRVPedigree, which allows researchers to simulate pedigrees ascertained on the basis of multiple, affected relatives. By incorporating the ascertainment process in the simulation, SimRVPedigree allows researchers to better understand the within-family patterns of relationship amongst affected individuals and ages of disease onset.

Conclusions: Through simulation, we show that affected members of a family segregating a rare disease variant tend to be more numerous and cluster in relationships more closely than those for sporadic disease. We also show that the family ascertainment process can lead to apparent anticipation in the age of onset. Finally, we use simulation to gain insight into the limit on the proportion of ascertained families segregating a causal variant. SimRVPedigree should be useful to investigators seeking insight into the family-based study design through simulation.
\end{abstract}

Keywords: Pedigree simulation, Family-based study, Rare variant, Ascertainment bias, Anticipation

\section{Background}

Family-based studies of pedigrees with multiple diseaseaffected relatives are regaining traction for identification of rare causal variants. These study designs were popular, for a time, but were eclipsed as genome-wide association studies (GWAS) gained popularity [1]. GWAS have been effective for identifying population associations with common variants genome-wide, but have low power to study rare variants [2]. Family-based studies require smaller sample sizes than their case/control counterparts and enjoy increased power to detect effects of rare variants [2]. Additionally, family-based studies are able to identify next-generation sequencing (NGS) errors by utilizing familial relationships to identify unlikely calls [2]. Improvements in the cost and technology associated with NGS have facilitated a revival in family-based studies [1]. Family-based analyses coupled with NGS can uncover rare variants that are undetected by GWAS [2]. For example, analysis of whole exome sequence data was used to

*Correspondence: jgraham@sfu.ca

${ }^{1}$ Department of Statistics and Actuarial Science, Simon Fraser University, 8888 University Drive, V5A 1S6, Burnaby, Canada

Full list of author information is available at the end of the article identify rare variants associated with non-syndromic oral clefts in large pedigrees ascertained to contain at least two affected relatives [3], to prioritize rare variants in large multi-generational pedigrees ascertained for multiple relatives diagnosed with bipolar disorder [4], and to identify rare variants segregating in families that contained at least two siblings with an autism spectrum disorder [5].

Unfortunately, family-based studies do not come without complication; for example, identifying a suitable number of pedigrees with desired criteria may be time consuming, sometimes requiring years to amass. In these circumstances, collecting new data to evaluate methodology or replicate findings is impractical. To address this challenge we have created an $R$ package, entitled SimRVPedigree, which simulates pedigrees ascertained to contain a minimum number of disease-affected relatives. SimRVPedigree models the affected individuals in an ascertained pedigree as the result of (1) sporadic disease or (2) a single, rare, diseasevariant segregating in the pedigree. At the individual level, SimRVPedigree models competing age-specific 
life events contingent on rare-variant status, disease status, and age through user supplied age-specific incidence rates of disease, and age-specific hazard rates for death. In a recursive manner, life events simulated at the individual level build and shape simulated pedigrees. Upon specification of user-defined study characteristics, SimRVPedigree will simulate pedigrees ascertained to contain multiple affected relatives according the specified criteria. To our knowledge, this is the only program to incorporate a competing risk model and account for the ascertainment process.

\section{Methods}

Given a sample of pedigrees we allow for the possibility that different families may segregate different rare variants, but assume that within a family genetic cases are due to a shared rare variant that increases disease susceptibility. We allow users to choose between two methods of rare variant introduction to the pedigree. One option is to assume that all ascertained pedigrees with genetic cases are segregating a variant that is rare enough to have been introduced by exactly one founder [6]. Alternatively, we allow users to simulate the starting founder's rare variant status with probability equal to the carrier probability of all causal variants considered as a group. When this option is selected some ascertained pedigrees may not segregate a causal variant. In either scenario, we assume that a causal variant is introduced by at most one founder and, when it is introduced, it is transmitted from parent to offspring according to Mendel's laws.

Starting at birth and ending with death, we simulate life events for the starting founder, censoring any events that occur after the last year of the study. We repeat this process, recursively, for all descendants of the founder allowing life events at the individual level to shape successive generations of the pedigree. To accomplish this, we condition on an individual's age, rare-variant status and disease status, and simulate waiting times to three competing life events: reproduction (i.e. producing offspring), disease onset, and death. We select the event with the shortest waiting time, update the individual's age by this waiting time, record the event type, and repeat this process from the new age until the individual dies or the end of the study is reached.

\section{Simulating life events}

To simulate life events SimRVPedigree users are required to specify:

hazardDF, a data frame of age-specific hazard rates, where column one represents the age-specific hazard rates for the disease in the general population, column two represents the age-specific hazard rates for death in the unaffected population, and column three represents the age-specific hazard rates for death in the affected population, and

partition, a discrete partition of ages over which to apply hazardDF.

Specifically, partition is a vector of ages, starting at age 0 , such that hazardDF $[k$,$] are the age-specific$ hazard rates for an individual whose age is contained in [partition [k], partition [k+1]). At the user's discretion, if the disease of interest is rare, the age-specific hazard rates for death in the unaffected population may be approximated by age-specific hazard rates for death in the general population. In the following subsections, we detail the procedures to simulate waiting times to onset, death, and reproductive events.

\section{Disease onset}

We model disease onset using a non-homogeneous Poisson process (e.g. [7]), conditioned on an individual's current age, $t^{\prime}$, rare-variant status, $x$, and disease status, $\delta$. In this context, $x=1$ if the individual is a carrier of the rare variant, and 0 otherwise; and $\delta=1$ if the individual has developed disease by age $t^{\prime}$, and 0 otherwise. Define $\kappa$ to be the relative-risk of disease for individuals who have inherited the causal variant and $\lambda_{o}(t)$ to be the baseline age-specific hazard rate of disease for an individual aged $t$ years. That is, $\lambda_{o}(t)$ is the age-specific hazard rate for individuals who do not carry a causal variant, i.e. sporadic cases. Let $\lambda_{\text {onset }}(t \mid x)$ denote the age-specific hazard rate of disease for an individual aged $t$ years conditioned on rare-variant status such that

$$
\lambda_{\text {onset }}(t \mid x)= \begin{cases}\lambda_{o}(t), & \text { if } x=0 ; \\ \kappa \cdot \lambda_{o}(t), & \text { if } x=1,\end{cases}
$$

for $\kappa \geq 1$.

If $p_{c}$ is the carrier probability of all causal variants considered as a group, then we can express the population age-specific hazard rate of disease, $\lambda_{\text {onset }}(t)$, as

$$
\lambda_{\text {onset }}(t)=\left(1-p_{c}\right) \lambda_{o}(t)+\kappa \cdot p_{c} \cdot \lambda_{o}(t) .
$$

Users are expected to provide $\lambda_{\text {onset }}(t)$; given $p_{c}$ and $\kappa$ we infer $\lambda_{o}(t)$ as $\lambda_{o}(t)=\frac{\lambda_{\text {onset }}(t)}{1+p_{c}(\kappa-1)}$. We note that this method for calculating $\lambda_{o}(t)$ has implications on the comparability of non-genetic individuals from studies simulated under very different $\kappa$ values. For example, when $p_{c}$ is constant, we see that for $\kappa_{1}<<\kappa_{2}$, the age-specific hazard rate for non-carrier individuals under genetic relative-risk $\kappa_{1}$ will be much greater than that of non-carrier individuals under genetic relative-risk $\kappa_{2}$. As $p_{c}$ increases this effect is visible more quickly for differing $\kappa$ values.

We note that not all individuals develop the disease; however, those who do are only permitted develop the disease once in our model. Individuals who have developed disease (i.e. $\delta=1$ ) do not develop disease again, but can 
reproduce or die. When $\delta=0$, we use intensity function $\lambda_{\text {onset }}(t \mid x)$ conditioned on rare-variant status, $x$, to simulate the waiting time to disease onset given current age, $t^{\prime}$. To clarify, if we denote the waiting time to disease onset by $W_{\text {onset }}$, and condition on the current age, $t^{\prime}$, the cumulative distribution function of $W_{\text {onset }}$ is given by

$P\left(W_{\text {onset }}<w \mid T=t^{\prime}, x\right)=1-\exp \left\{-\int_{t^{\prime}}^{t^{\prime}+w} \lambda_{\text {onset }}(u \mid x) d u\right\}$.

\section{Death}

We model death using a non-homogeneous Poisson process, conditioned on an individual's current age, $t^{\prime}$, and disease status, $\delta$. Define $\delta$ as in the previous discussion, and let $\lambda_{u}(t)$ and $\lambda_{a}(t)$ denote the age-specific hazard rates of death, for individuals aged $t$ years, in the unaffected population and the affected population, respectively. We use intensity function $\lambda_{\text {death }}(t \mid \delta)$ conditioned on disease status $\delta$ to simulate the waiting time to death given the current age, $t^{\prime}$. In this context, $\lambda_{\text {death }}(t \mid \delta)$ represents the age-specific hazard rate of death for an individual aged $t$ years conditioned on their disease status, which we model as

$$
\lambda_{\text {death }}(t \mid \delta)=\left\{\begin{array}{l}
\lambda_{u}(t), \text { if } \delta=0 \\
\lambda_{a}(t), \text { if } \delta=1
\end{array}\right.
$$

We do not model disease remission; after an individual has developed disease we use the age-specific hazard rates for death in the affected population to model their waiting time to death.

\section{Reproduction}

To accommodate extra-Poisson variability in the number of human offspring, we use a negative-binomial model with number of trials $n \approx 2$ and success probability $p \approx$ $4 / 7$, as proposed by [8]. We adopt this negative-binomial model of offspring number in SimRVPedigree. We employ an equivalent Poisson-Gamma mixture model [9] to obtain the negative-binomial offspring number and to simulate the waiting time to reproduction.

Let $w_{t^{\prime}}$ denote the waiting time to reproduction given an individual's current age $t^{\prime}$, and assume that simulated subjects are able to reproduce from age $a_{1}$ to age $a_{2}$. To mimic observed data on first-born live births (see Additional file 1: Section 6), we simulate $a_{1}$ and $a_{2}$ as follows: sample $a_{1}$ uniformly from ages 16 to 27 , and $a_{2}-a_{1}$ uniformly from 10 to 18 years. At birth we simulate an individual's lifetime birthrate by taking a random draw, $\gamma$, from a gamma distribution with shape 2 and scale 4/3. Individuals who draw large $\gamma$ will have high birth rates and many children, whereas individuals who draw small $\gamma$ will have low birth rates and few or no children.

For some diseases, users may want to reduce the birth rate after disease onset; we allow users to achieve this through an additional parameter $f$, assumed to be between
0 and 1 , which is used to rescale the birth rate after disease onset. By default, $f=1$ so that the birth rate remains unchanged after disease onset. Given an individual's birth rate, current age, and disease status, $\delta$, we obtain their waiting time to reproduction as follows:

1 Simulate the unconditional waiting time to reproduction by drawing $w$ from an exponential distribution with rate $\frac{\gamma f \delta+\gamma(1-\delta)}{\left(a_{2}-a_{1}\right)}$.

2 Condition on the current age, $t^{\prime}$, to obtain the conditional waiting time to reproduction:

$$
w_{t^{\prime}}= \begin{cases}a_{1}+w-t^{\prime}, & \text { if } t^{\prime}<a_{1} \text { and }\left(a_{1}+w\right)<a_{2} ; \\ t^{\prime}+w, & \text { if } t^{\prime} \in\left[a_{1}, a_{2}\right) \text { and }\left(t^{\prime}+w\right)<a_{2} ; \\ \infty, & \text { otherwise. }\end{cases}
$$

\section{Pedigree simulation}

To simulate all life events for a subject, starting at birth we generate waiting times to disease onset, death, and reproduction, as outlined previously and choose the event with the shortest waiting time to be the next life event. Next, we add the waiting time associated with the earliest event to the current age and either record the year of disease onset or death, or add a new offspring to the pedigree. We repeat this process from the updated age, recursively, until the individual dies or the study stop year is reached. This algorithm details the full life event procedure at the individual level. Complete details are available in Additional file 1.

To simulate a full pedigree, we recursively apply the algorithm described above, as follows:

- Step 1: Simulate life events for the first founder given rare-variant status.

- Step 2: Simulate life events for any new offspring given rare-variant status as outlined above.

- Step 3: Repeat step 2 until life events have been simulated for all offspring.

\section{Ascertainment features}

The primary function of SimRVPedigree, sim_RVped ( ), simulates pedigrees ascertained for multiple disease-affected relatives. We allow users to specify family-based study features through the following arguments of sim_RVped ( ) :

num affected: the minimum number of diseaseaffected relatives required for ascertainment of the pedigree.

ascertain_span: the start and stop year for pedigree ascertainment.

stop_year: the last year of follow-up for the pedigree.

recall_probs: the proband's recall probabilities for relatives of varying degree. 
In this context, the proband is the affected family member first in contact with the study, presumably at the time of disease onset.

The ascertainment span represents the time span, in years, over which the family could be ascertained through the proband. For example, suppose that a particular study ascertained families, containing at least two affected members, from 2000 to 2010. In this scenario, the user would set ascertain_span $=c(2000$, 2010) and num_affected $=2$. The sim_RVped () function would then simulate families such that the proband developed disease between 2000 and 2010 and was at least the second family member to develop disease.

The study stop year represents the last year data are collected for ascertained families. Consider the previous study, and suppose that data were collected until 2016. To achieve this in simulation, users would simply specify stop_year $=2016$, which would result in sim_RVped () simulating life events for ascertained families until the year 2016.

Often researchers involved in family-based studies are confronted by incomplete ascertainment of a proband's relatives, which could occur if the proband cannot provide a complete family history, or if he or she does not support contact of specific relatives. SimRVPedigree allows users to mimic this scenario, in simulation, by trimming relatives from a pedigree based on the proband's probability of recalling them. To specify a proband's recall probabilities for his or her relatives, i.e. recall_probs, the user provides a list of length $q$, such as $p=\left(p_{1}, p_{2}, \ldots, p_{q}\right)$. In this context, $p_{i}$ is used to denote the proband's recall probability for a relative of degree $i$ when $i=1,2, \ldots, q-1$, or the proband's recall probability for a relative of degree $q$ or greater when $i=q$. To simulate fully ascertained families, we set recall_probs = C (1), which corresponds to $p=1$. Alternatively, if unspecified, recall_probs is set to four times the kinship coefficient, e.g. [10]. This default value retains the proband's first-degree relatives (i.e. parents, siblings, and offspring) with probability 1 , second-degree relatives (i.e. grandparents, grandchildren, aunts, uncles, nieces, and nephews) with probability 0.5 , third-degree relatives with probability 0.25 , etc.

In the event that a trimmed relative is required to fully specify the relationships among recalled family members, we include the trimmed relative, mark them as unavailable, and remove (i.e. mark as missing) any of their relevant information. That is, disease status, relative-risk of disease, and event years are all missing for any relatives not recalled by the proband. Since disease-affected relatives may be trimmed from a pedigree, trimmed pedigrees may contain fewer than num affected diseaseaffected relatives. When this occurs, sim_RVped() will discard the pedigree and simulate another until all conditions specified by the user are met.

\section{Results}

\section{Settings}

In the following applications, we use SimRVPedigree in conjunction with $\mathrm{R}$ [11] to investigate the effect of the relative-risk of disease in genetic cases, $\kappa$, on ascertained pedigrees. We first investigate the effect of $\kappa$ on the number of affected relatives per family, and on the degree of familial clustering among affected relatives. Next, we investigate how ages of onset from more recent generations tend to be younger than those from older generations in the ascertained pedigrees [12], a phenomenon which we refer to as apparent anticipation. Lastly, we demonstrate how SimRVPedigree may be used to estimate the proportion of families that segregate the causal variant in a sample of ascertained pedigrees.

To study pedigrees ascertained to contain multiple relatives affected by a lymphoid cancer, we simulated study samples according to the following criteria.

1 Each study sample contained a total of one thousand pedigrees, ascertained from the year 2000 to the year 2015.

2 Each pedigree contained at least two relatives affected by lymphoid cancer.

3 The birth year of the founder who introduced the rare variant to the pedigree was distributed uniformly from 1900 to 1980.

4 For each $\kappa$ considered, the carrier probability, $p_{c}$, for all causal variants with genetic-relative risk $\kappa$ was assumed to be 0.002 .

5 Sporadic cases, i.e. affected individuals who did not inherit the rare variant, develop lymphoid cancer according to the baseline, age-specific hazard rate of lymphoid cancer. The population, age-specific hazard rate of lymphoid cancer were estimated through the Surveillance, Epidemiology, and End Results (SEER) Program [13, 14], and are displayed in Fig. 1.

6 Genetic cases, i.e. affected individuals who did inherit the rare variant, develop lymphoid cancer at $\kappa$ times the baseline, age-specific hazard rate of lymphoid cancer. We considered $\kappa \in(1,10,20)$ and simulated one thousand pedigrees for each $\kappa$ considered.

7 Since lymphoid cancer accounts for a relatively small proportion of all deaths, the age-specific hazard rate for death in the unaffected population was approximated by that of the general population. Individuals who do not develop lymphoid cancer die according to the age-specific hazard rate of death in the general population [15], while individuals who have developed lymphoid cancer die according to the age-specific hazard rate of death in the affected 

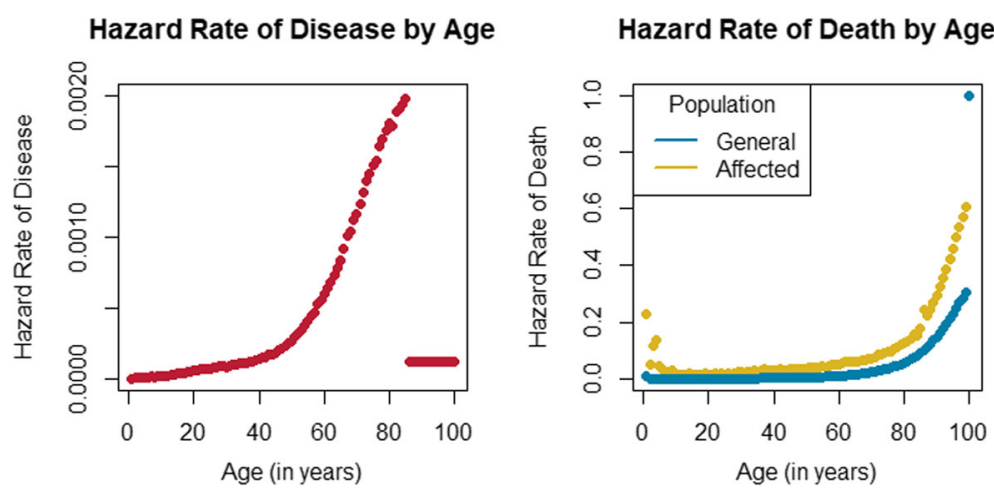

Fig. 1 Hazard Rates. (Left) Baseline, age-specific hazard rates of lymphoid cancer estimated by SEER [13, 14]. SEER provides age-specific incidence and morality data, in yearly increments, up to age 84 years, and then aggregates data for ages of 85 years or greater. We considered the SEER reported incidence rate for individuals of age 85 or greater to be the constant hazard rate of disease for individuals between the ages of 85 to 100 . (Right) Age-specific hazard rates of death for the general population [15] and for the disease-affected population [13, 16, 17]. To promote continuity in the age-specific hazard rate of death for the affected population, we assume that it is twice that of the unaffected population after age 84 years. After age 84 years, the SEER data do not allow for the age-specific hazard rates of death in the affected population to be estimated in yearly increments

population $[13,16,17]$. Figure 1 displays the age-specific hazard rates of death for these two groups.

8 The proband's probabilities for recalling relatives were set to recall_probs $=(1,1,1$, $0.5,0.125)$, so that all first, second, and third-degree relatives of the proband were recalled with probability 1 , all fourth-degree relatives of the proband were recalled with probability 0.5 , and all other relatives of the proband were recalled with probability 0.125 .

9 The stop year of the study was set to 2017.

\section{Example}

We demonstrate how to simulate a single pedigree according to the settings described previously.

After installing SimRVPedigree, we load the package in $\mathrm{R}$ using the library function.

R> library (SimRVPedigree)

Suppose that we can obtain age-specific hazard rates in yearly increments starting at age 0 and ending with age 100. In this case, we define the partition of ages over which to apply the age-specific hazards rates using the seq function.

$\mathrm{R}>$ age_part <- $\operatorname{seq}(0,100$, by $=1)$

Next, assume that LC_Hazards is a data frame whose columns provide age-specific hazard rates, in yearly increments, from age 0 to age 100 , as indicated below.

LC_Hazards [, 1] Age-specific hazard rates of lymphoid cancer in the general population.

LC_Hazards [, 2] Age-specific hazard rates of death for individuals in the general population.

LC_Hazards [, 3] Age-specific hazard rates of death for individuals who have lymphoid cancer.
We create a new object of class hazard from the partition of ages, age part, and the data frame of hazard rates, LC_Hazards, by executing the following command.

$$
\begin{array}{r}
\text { R> haz_mat <- hazard (partition = age_part, } \\
\text { hazardDF }=\text { LC_Hazards) }
\end{array}
$$

To simulate a single pedigree with family identification number 1 and a genetic relative-risk of 10, assuming that the eldest founder introduces the variant, and according to the settings described previously we use the following command.

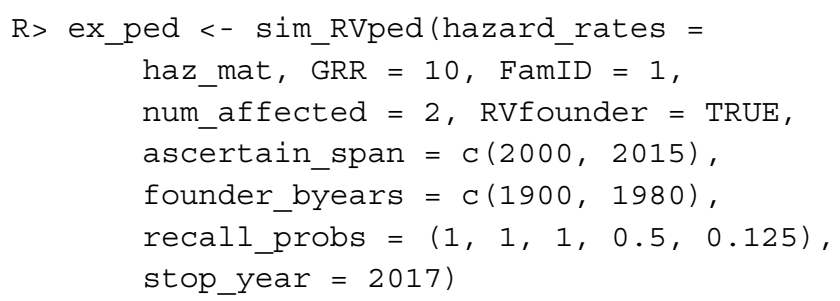

To view a description of the contents of ex_ped we use the summary command.

R> summary (ex_ped)

$\begin{array}{lrrr} & \text { length } & \text { Class } & \text { Mode } \\ \text { full_ped } & 15 & \text { ped } & \text { list } \\ \text { ascertained_ped } & 15 & \text { ped } & \text { list }\end{array}$

Upon executing the command above, we see that ex_ped is a list containing two objects of class ped. The first is named full_ped and represents the original pedigree, prior to proband selection and trimming. The second is named ascertained ped and represents the ascertained pedigree; this data frame includes an additional variable to identify the proband. In this application, we are interested in families that were ascertained for study; hence, we focus attention on ascertained_ped.

To simplify the following examples, we store the ascertained pedigree as study_ped. 
R> study_ped <- ex_ped\$ascertained_ped

To plot the ascertained pedigree we simply supply the pedigree to the plot function.

R> plot (study_ped)

The plotted pedigree is displayed in Fig. 2.

To obtain summary information for study_ped we supply it to summary.

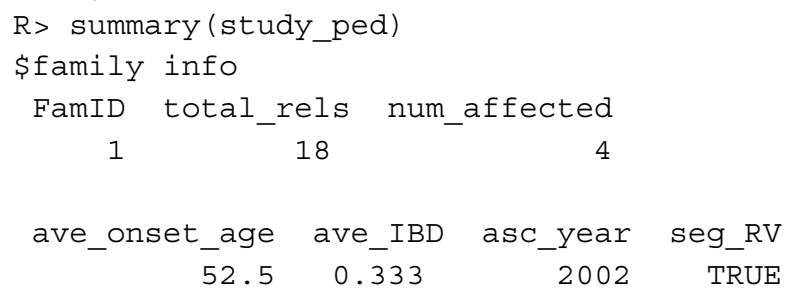

As displayed above, when the argument of summary is an object of class ped, summary returns two data frames named family_info and affected_info.
The family_info data frame catalogues the information for the entire family. For each family supplied it provides (from left to right): family identification number, the total number of relatives in the pedigree, the total number of disease-affected relatives in the pedigree, the average onset age of the disease-affected relatives, the average of the pairwise probabilities of identity by descent (IBD) among the disease-affected relatives in the pedigree, the ascertainment year of the pedigree, and a logical variable indicating whether or not the pedigree segregates a casual variant. The affected_info data frame catalogues information for the disease-affected relatives. For each disease-affected relative it details (from left to right): family identification number, individual identification number, year of birth, year of disease-onset, year of death, relative risk of disease, proband status, and rare variant status.

\section{Applications}

\section{Number of disease-affected relatives}

To illustrate how the number of disease-affected relatives in each pedigree varies with $\kappa$, we refer to the data described in Settings. This data contains simulated study samples, containing 1000 pedigrees, for $\kappa=1, \kappa=10$, and $\kappa=20$.

Figure 3 summarizes the distribution of the number of disease-affected relatives per pedigree for these three groups. From the figure we see that for $\kappa=1$ this distribution is more highly concentrated at two affected members than for the other two groups considered. Not

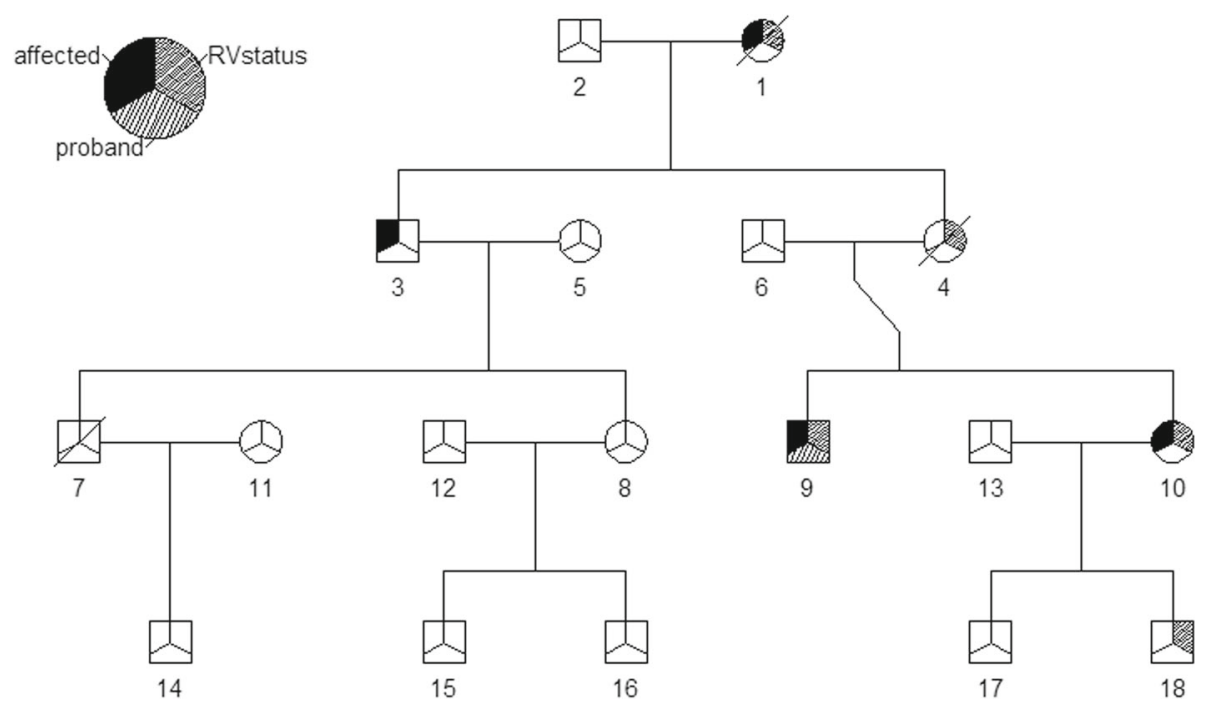

Fig. 2 Simulated Pedigree. In this pedigree squares are used to symbolize males and circles are used to symbolize females. Mates are connected by a horizontal line, and their offspring branch out below. Individuals who have died have a slash through their symbol. As indicated by the legend, if the upper left third of an individual's symbol is shaded black, then that individual is disease-affected. If the upper right third of an individual's symbol is shaded, then that individual is a carrier of the causal variant. If the bottom third of an individual's symbol is shaded, then that individual is the proband 


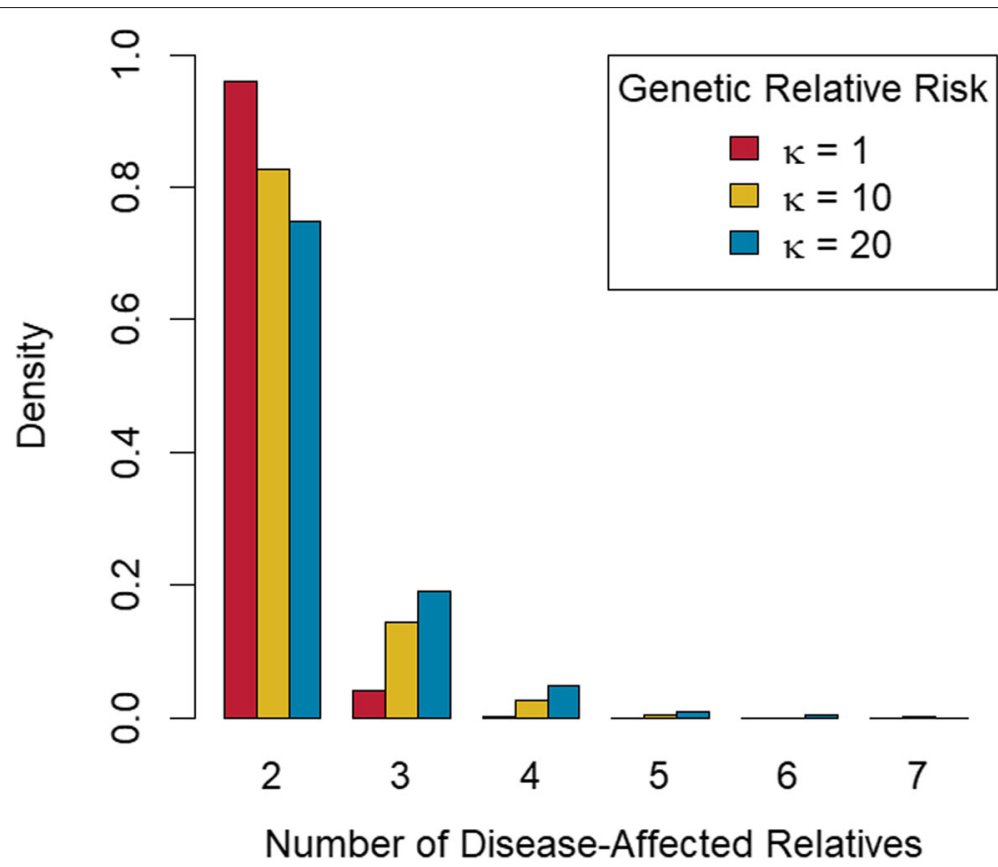

Fig. 3 Bar charts of Number of Disease-Affected Relatives per Pedigree. Barcharts of number of disease-affected relative per pedigree grouped by genetic relative-risk of disease, $\kappa$

surprisingly, as $\kappa$ increases we see relatively fewer families containing only two affected members, and more families containing three or greater affected members.

\section{Familial clustering}

To investigate the relationship between familial clustering among affected relatives and $\kappa$, we restrict attention to pedigrees that contained two or three affected relatives. We did not consider pedigrees with four or more disease-affected relatives because these pedigrees are rarely observed when $\kappa=1$. This resulted in a total of 999 simulated pedigrees in the $\kappa=1$ group, 970 simulated pedigrees in the $\kappa=10$ group, and 939 simulated pedigrees in the $\kappa=20$ group. To assess the level of familial clustering among affected relatives, we computed the average of the pairwise IBD probabilities among affected members in a pedigree, which we will denote by $\mathcal{A}_{I B D} . \mathcal{A}_{I B D}$ is proportional to the genealogical index of familiality statistic [18], which has been used to summarize familial clustering of aggressive prostate cancer in the Utah population. In general, the IBD probability between two relatives decreases as they become more distantly related. For example, for an affected parent-child pair, or two affected siblings $\mathcal{A}_{I B D}=0.5$; whereas for an affected avuncular pair, or an affected grandparent-grandchild pair $\mathcal{A}_{\text {IBD }}=0.25$.

Figure 4 shows the conditional distribution of $\mathcal{A}_{I B D}$ given the total number of affected relatives in a pedigree and $\kappa$. Tabulated results for Fig. 4 are available in Additional file 1: Section 2. The left panel of Fig. 4 summarizes the conditional distribution of $\mathcal{A}_{I B D}$ for families with two affected members. The conditional distribution of $\mathcal{A}_{I B D}$ shifts probability mass toward 0.5 as $\kappa$ increases and suggests that disease-affected individuals tend to be more closely related in families with larger values of $\kappa$. The right panel of Fig. 4 summarizes the conditional distribution of $\mathcal{A}_{I B D}$ among families with three affected members, and shows the same trend as the left panel, of $\mathcal{A}_{I B D}$ values shifted towards 0.5 for larger values of $\kappa$.

\section{Anticipation}

Anticipation is a decreasing trend in the age of disease onset, and possibly an increasing trend in severity, in successive generations of a family [19]. Some genetic diseases with unstable repeat expansions show anticipation, and include: Huntington's Disease, fragile X syndrome, and myotonic dystrophy [20].

However, studies of genetic anticipation based solely on the ages of onset of affected members have the potential for ascertainment bias [21]. Possible sources of ascertainment bias include: early detection in offspring due to parental diagnosis or improved diagnostic techniques and right-censoring of family members who have developed the disease by the end of the study, especially in studies of large multi-generational pedigrees that have been ascertained to contain multiple affected members. $[12,21]$.

Referring to the data described in section Settings, we illustrate how apparent anticipation can arise as an 

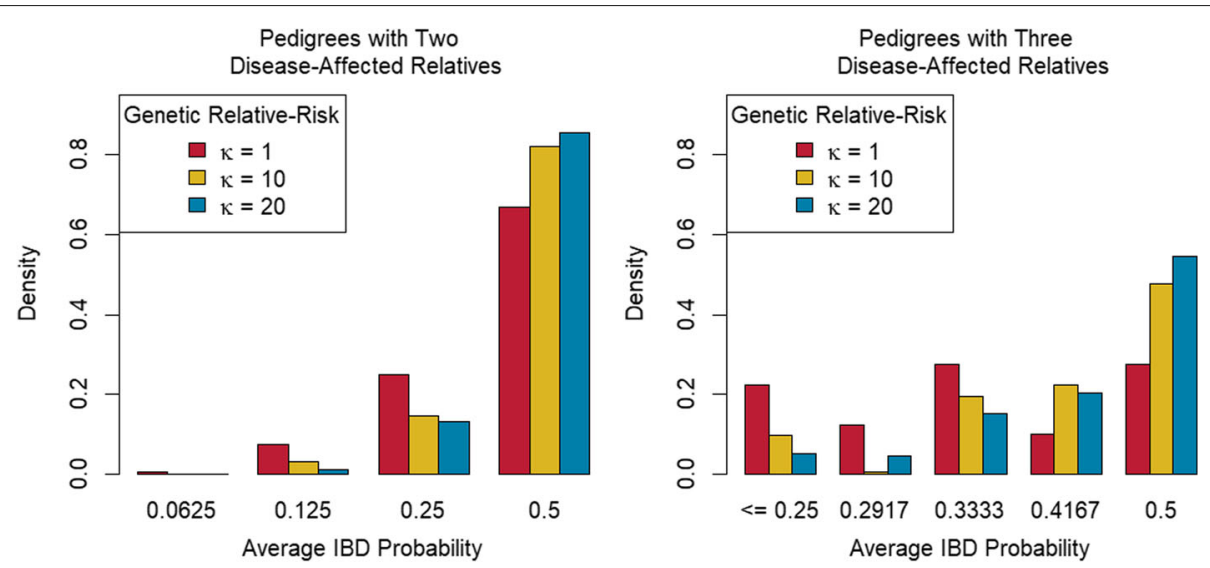

Fig. 4 Bar charts of $\mathcal{A}_{I B D}$ Distributions. Barcharts of $\mathcal{A}_{B D D}$ distributions for pedigrees with two (left) or three (right) disease-affected relatives, grouped by genetic relative-risk of disease

artefact of studies ascertaining families with multiple disease-affected relatives. Within each of the families considered, generation number was assigned among affected relatives so that generation number one represents the most recent common ancestor with whom all affected members could share a variant identical by descent. In this assignment scheme, we allow an affected individual to be his or her own most recent common ancestor. To demonstrate this convention, consider a family with two affected relatives: if the affected members are a parent-child pair, then the parent would be assigned generation number one, and the child assigned generation number two. However, if the affected members are a sibling pair, each sibling would be assigned generation number two, since a parent is the closest relative from whom the affected siblings could have inherited a disease variant.

Figure 5 displays the ages of onset, by assigned generation, grouped by $\kappa$, the relative-risk of disease for genetic cases. We emphasize that SimRVPedigree does not include a mechanism to simulate anticipation. However, we note that even though anticipation is not present in the simulated data, within each genetic-relative-risk group considered, the box plots exhibit a decreasing trend in the ages of onset for successive generations. The false anticipation signal is likely due to many of the ascertained pedigrees being large, and multi-generational, and therefore prone to right-censoring of younger family members who will develop disease later in life, after the study stop year.

If there is right-censoring of younger family members then this censoring should be apparent in their ages of death as well. Therefore it is useful to consider using the ages of death in unaffected relatives as a negative control to gain insight into ascertainment bias [19]. Box plots of the ages of death in unaffected relatives by generation for the relative-risk groups are similar to those in
Fig. 5 for the age of onset in disease-affected relatives. This similarity strongly suggests the presence of ascertainment bias. Further details of this investigation may be found in Additional file 1: Section 3.

\section{Proportion of ascertained pedigrees segregating a causal variant}

Familial lymphoid cancer, i.e. a family that contains multiple relatives affected by lymphoid cancer, is relatively rare; however, lymphoid cancer is not a rare disease as it affects roughly 1 in 25 [13, 14]. With such diseases, there is a greater risk of ascertaining pedigrees that contain multiple disease-affected relatives by chance alone. Since we do not expect these pedigrees to segregate a causal variant it is advantageous to choose ascertainment criteria that reduces the likelihood of sampling such pedigrees.

To determine what proportion of ascertained families we expect to segregate a causal variant we conducted a simulation study in which the rare variant status of the starting founder was allowed to vary so that fully sporadic pedigrees were given an opportunity for ascertainment.

The procedure to simulate a study containing both genetic and sporadic families may be described as follows.

Step 1: Allow the starting founder to introduce a causal variant with genetic relative-risk $\kappa$ with probability 0.002 .

Step 2: Simulate the rest of the pedigree, according to the settings described in Settings, and add it to our sample of ascertained pedigrees if it meets the ascertainment criteria.

Step 3: Repeat steps one and two until the requisite number of pedigrees have been ascertained.

For this procedure we considered $\kappa=1$ and all multiples of 5 between 5 and 100 , i.e. $\kappa \in(1,5,10,15, \ldots, 95,100)$. For each $\kappa$ considered we simulated a family study 


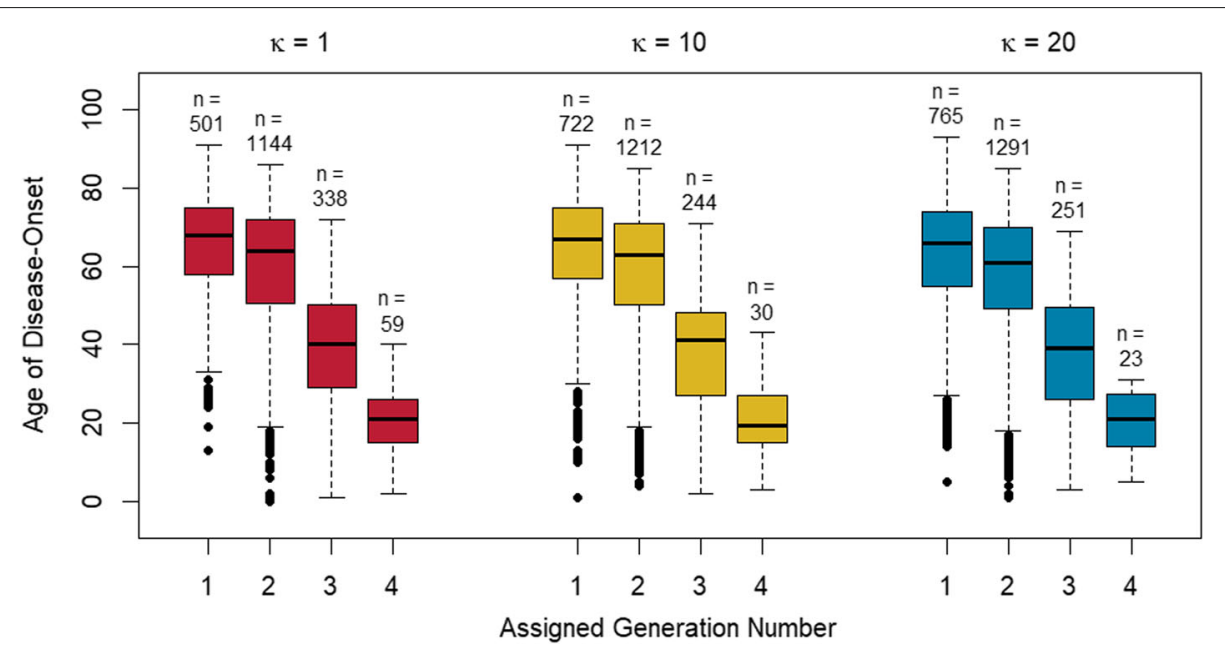

Fig. 5 Box plots of Age of Disease Onset by Assigned Generation Number. Boxplots of age of onset by assigned generation number, as defined in text, grouped by genetic relative-risk of disease, $\kappa$. The numbers of observations, $n$, used to create each box plot are displayed above their respective plots

containing one thousand ascertained pedigrees. Next, we determined what proportion of the ascertained pedigrees were segregating a causal variant that increased disease susceptibility. The results of this investigation are displayed in Fig. 6. The leftmost panel in Fig. 6 indicates that most of the ascertained pedigrees are not segregating a causal variant. For example, when the genetic relativerisk is 20 , we see that less than $20 \%$ of the ascertained pedigrees with two or more disease-affected relatives are segregating a causal variant. Focusing attention on the ascertained pedigrees that contain three or more affected relatives (the middle panel of Fig. 6) we see that these pedigrees tend to segregate a causal variant more often than the pedigrees that only contained two or more affected relatives. When we restrict our focus to the ascertained pedigrees that contain four or more affected relatives (the rightmost panel of Fig. 6), we see more of these pedigrees tend to segregate a causal variant. These estimates tend to be more erratic because we don't often observe fully sporadic families with four or more affected relatives. Among the original samples of one thousand pedigrees, we observe only two fully sporadic pedigrees with five affected relatives, and none with six or more disease-affected relatives.

These results indicate that when a disease is not rare, and when the carrier probability of the causal variant is very low (i.e. $p_{c}=0.002$ ), focusing on families with at least three affected relatives is more effective for sampling pedigrees that segregate a causal variant. Focusing on pedigrees with

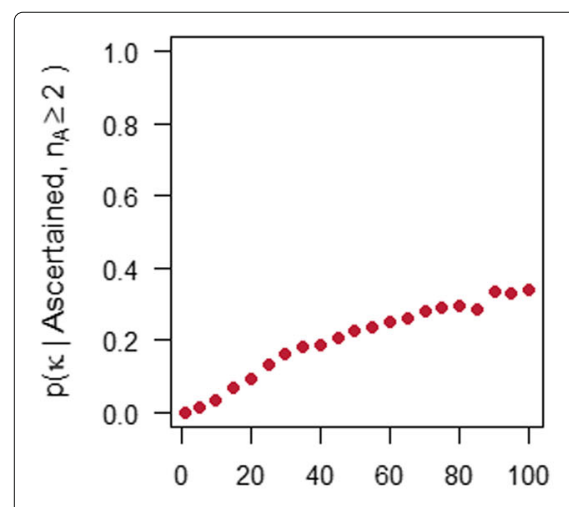

Genetic Relative-Risk, $\kappa$

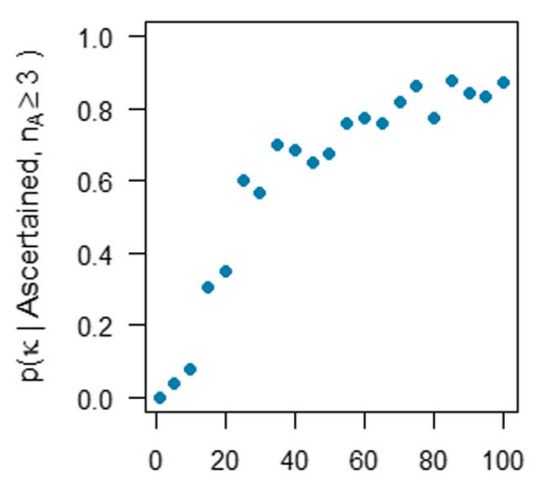

Genetic Relative-Risk, $\kappa$

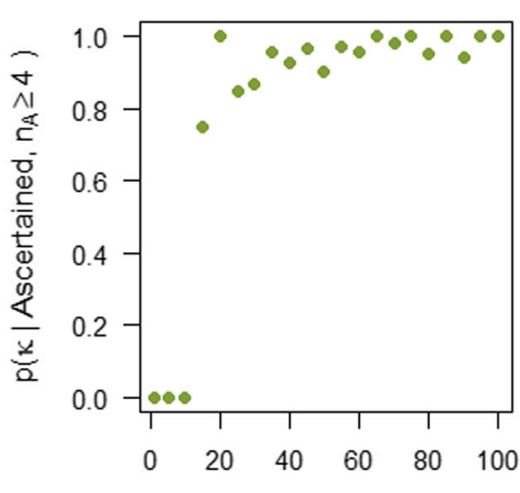

Genetic Relative-Risk, $\kappa$

Fig. 6 Genetic Contribution Estimate. Scatter plots of the probability that a randomly selected pedigree from a sample of ascertained pedigrees is segregating a genetic variant with relative-risk of disease $\kappa$ against the relative-risk of disease $\kappa$. Here we consider the effect of restricting attention to the ascertained pedigrees with $n_{A}$ or more disease-affected relatives. In the leftmost panel, we consider all one thousand pedigrees ascertained with two or more disease-affected relatives; in the middle panel, we consider the subset with three or more disease-affected relatives, and in the right most panel the subset with four or more disease-affected relatives 
at least four affected relatives provides even greater improvement.

\section{Computation time}

We would like to note that simulation of ascertained pedigrees can be computationally expensive. Therefore, we urge users to take advantage of parallel processing, in R, or cluster computing when simulating a large number of ascertained pedigrees.

There are several factors that effect the amount of time required to simulate a pedigree. For example, the genetic relative-risk, the probability that a causal rare variant is segregating in the family, and the ascertainment span, to name a few. To illustrate the effect of the genetic relativerisk on timing we consider the family-study described in Settings. The following table provides summary statistics for the average computation time, in seconds, required to simulate a single pedigree on a Windows OS with an i7$4790 @ 3.60 \mathrm{GHz}, 12$ GB of RAM, and a C220 SATA AHCI (Table 1).

When probability that a causal rare variant is segregating in the family is small, the simulation time will tend towards the time required to simulate an ascertained pedigree with a genetic relative-risk of 1 . This is the case for all pedigrees simulated in Proportion of ascertained pedigrees segregating a causal variant since the probability that the eldest founder introduces the rare variant is 0.002 .

\section{Discussion}

We provide several applications for SimRVPedigree to illustrate the effect of the genetic relative-risk, $\kappa$, on features of the ascertained pedigrees. First, we investigate the relationship between $\kappa$ and the number of affected individuals in each ascertained family. In this application, as $\kappa$ increases we observe pedigrees that contain three or more affected relatives more frequently than pedigrees with only two affected relatives.

Second, we examine the relationship between $\kappa$ and the average, pairwise IBD probability among affected relatives in a pedigree. We observe that pedigrees simulated with larger values of $\kappa$ tend to contain affected relatives that are more closely-related than pedigrees simulated with smaller values of $\kappa$.

Table 1 Comparison of Computation Time for Various Genetic Relative-Risk Values

\begin{tabular}{llll}
\hline $\begin{array}{l}\text { Genetic } \\
\text { relative-risk }\end{array}$ & $\begin{array}{l}\text { Average simulation } \\
\text { time (in seconds) }\end{array}$ & $\begin{array}{l}\text { Standard deviation } \\
\text { of simulation time } \\
\text { (in seconds) }\end{array}$ & $\begin{array}{l}\text { Number } \\
\text { of trials }\end{array}$ \\
\hline 1 & 30.752 & 32.539 & 50 \\
10 & 1.461 & 1.339 & 50 \\
20 & 0.650 & 0.564 & 50 \\
\hline
\end{tabular}

Tabulated average computation time and standard deviation of computation time, in seconds. These results were obtained over 50 repeated simulations of a single pedigree
Third, we illustrate that the family-based study design can contribute to apparent anticipation signals. In part, this is due to large, multi-generational pedigrees, which are prone to right-censoring of younger family members likely to experience disease onset later in life. This type of right-censoring can confound true genetic anticipation. We observe that it is possible to reduce this bias by following family members available at the time of ascertainment for a sufficient length of time. However, the necessary time frame (roughly 100 years) is impractical for real studies [see Additional file 1: Section 4].

Finally, we show how users can estimate the proportion of ascertained pedigrees that are segregating a variant that increases disease susceptibility. In this application we find that when the carrier probability of all causal variants considered as a group is 0.002 , many of the pedigrees ascertained with two or more disease-affected relatives do not segregate a genetic variant. In this scenario, it may be advantageous for researchers to focus on pedigrees with three or more disease-affected relatives. We note that when the carrier probability increases results will vary [see Additional file 1: Section 5]. SimRVPedigree is intended for simulating diseases that are influenced by rare variants (e.g. allele frequency $<0.005$ ); however, when the carrier probability is increased to reflect variants that are less rare (e.g. allele frequency $\in[0.005,0.01]$ ), SimRVPedigree may underestimate the proportion of ascertained pedigrees that contain genetic cases.

We emphasize that ascertained families can differ substantially depending on the simulation settings chosen. For example, variations in the ascertainment span can affect the distribution of the number of affected relatives in each pedigree, when all other study settings remain constant.

\section{Conclusions}

The SimRVPedigree package provides methods to simulate pedigrees that contain multiple disease-affected relatives ascertained by a family-based study. To simulate life events at the individual level, SimRVPedigree models disease onset, death, and reproduction as competing life events; thus, pedigrees are shaped by the events simulated at the individual level. SimRVPedigree allows for flexible modelling of disease onset through user-supplied age-specific hazard rates for disease onset and death, and also permits flexibility in family-based ascertainment.

Among their benefits, family-based studies of large pedigrees with multiple disease-affected relatives enjoy increased power to detect effects of rare variants [2]. However, to conduct a family-based study of a rare disease it may take years to collect enough data. For planning and inference, we present the SimRVPedigree package to readily simulate pedigrees ascertained for multiple relatives affected by a rare disease. To our knowledge, this is the first package to dynamically simulate pedigrees to account for competing life events. 


\section{Additional file}

Additional file 1: SimRVPedigree Supplement. This is a pdf file that provides detailed information about the simulation procedure, as well as additional information for the applications discussed in the main text. (PDF $254 \mathrm{~kb}$ )

\section{Abbreviations}

GWAS: Genome-wide association studies; IBD: Identity by descent; NGS: Next-generation sequencing

\section{Funding}

This work was supported in part by the Natural Science and Engineering Research Council of Canada (NSERC), the Canadian Institutes of Health Research (CIHR), and the Canadian Statistical Sciences Institute (CANSSI).

\section{Availability of data and materials}

SimRVPedigree is a platform-independent package and is readily available for $R \geq 3.4 .0$ through the CRAN repository. SimRVPedigree requires kinship2 $\geq 1.6 .4$ and dplyr $\geq 0.7 .4$.

The datasets generated and/or analysed during the current study are available in the SimRVPedigree-Supplementary-Data repository, https://github.com/ cnieuwoudt/SimRVPedigree---Supplementary-Data.

\section{Authors' contributions}

CN developed the R package, drafted the manuscript and conducted the analyses. SJ and ABW provided the initial motivation for the project and genetics guidance. JG conceived of the R package and the overall statistical approach, and contributed to the development of the manuscript and the applications. All authors read and approved the final manuscript.

\section{Ethics approval and consent to participate}

Not applicable.

\section{Consent for publication}

Not applicable.

\section{Competing interests}

The authors declare they have no competing interests.

\section{Publisher's Note}

Springer Nature remains neutral with regard to jurisdictional claims in published maps and institutional affiliations.

\begin{abstract}
Author details
${ }^{1}$ Department of Statistics and Actuarial Science, Simon Fraser University, 8888 University Drive, V5A 1S6, Burnaby, Canada. ${ }^{2}$ Canada's Michael Smith Genome Sciences Centre, British Columbia Cancer Agency, 675 W 10th Ave, V5Z 1L3, Vancouver, Canada. ${ }^{3}$ Department of Medical Genetics, University of British Columbia, Vancouver, Canada. ${ }^{4}$ Department of Biomedical Physiology and Kinesiology, Simon Fraser University, 8888 University Drive, V5A 1S6, Burnaby, Canada.
\end{abstract}

Received: 22 January 2018 Accepted: 2 October 2018

Published online: 15 October 2018

\section{References}

1. Thomas DC. Some surprising twists on the road to discovering the contribution of rare variants to complex diseases. Hum Hered. 2012 https://doi.org/10.1159/000347020.

2. Wijsman EM. The role of large pedigrees in an era of high-throughput sequencing. Hum Genet. 2012. https://doi.org/10.1007/s00439-012 1190-2.

3. Bureau A, Parker MM, Ruczinski I, Taub MA, Marazita ML, Murray JC, Mangold E, Noethen MM, Ludwig KU, Hetmanski JB, Bailey-Wilson JE, Cropp CD, Li Q, Szymczak S, Albacha-Hejazi H, Alqosayer K, Field LL, Wu-Chou YH, Doheny KF, Ling H, Scott AF, Beaty TH. Whole exome sequencing of distant relatives in multiplex families implicates rare variants in candidate genes for oral clefts. Genetics. 2014. https://doi.org/ 10.1534/genetics.114.165225
4. Cruceanu C, Ambalavanan A, Spiegelman D, Gauthier J, Lafrenière RG Dion PA, Alda M, Turecki G, Rouleau GA. Family-based exome-sequencing approach identifies rare susceptibility variants for lithium-responsive bipolar disorder. Genome. 2013. https://doi.org/10. 1139/gen-2013-0081.

5. Toma C, Torrico B, Hervás A, Valdés-Mas R, Tristán-Noguero A, Padillo V, Maristany M, Salgado M, Arenas C, Puente XS, Bayés M, Cormand B. Exome sequencing in multiplex autism families suggests a major role for heterozygous truncating mutations. Mol Psychiatry. 2013. https://doi.org/ 10.1038/mp.2013.106

6. Bureau A, Younkin SG, Parker MM, Bailey-Wilson JE, Marazita ML, Murray JC, Mangold E, Albacha-Hejazi H, Beaty TH, Ruczinski I. Inferring rare disease risk variants based on exact probabilities of sharing by multiple affected relatives. Bioinformatics. 2014. https://doi.org/10.1093/ bioinformatics/btu198.

7. Gallager RG. Poisson processes. In: Discrete Stochastic Processes. Boston: Springer; 1996. p. 31-55.

8. Kojima Kl, Kelleher TM. Survival of mutant genes. Am Nat. 1962. https:// doi.org/10.1086/282242.

9. Zhou M, Carin L. Negative binomial process count and mixture modeling. IEEE Trans Pattern Anal Mach Intell. 2015. https://doi.org/10. 1109/tpami.2013.211.

10. Thompson EA. Statistical Inference from Genetic Data on Pedigrees. Beachwood: Institute of Mathematical Statistics; 2000.

11. R Core Team: R: A Language and Environment for Statistical Computing. Vienna: R Foundation for Statistical Computing; 2017. R Foundation for Statistical Computing. https://www.R-project.org/.

12. Ridley RM, Frith CD, Crow TJ, Conneally PM. Anticipation in huntington's disease is inherited through the male line but may originate in the female. J Med Genet. 1988. https://doi.org/10.1136/jmg.25.9.589.

13. Surveillance Research Program: National Cancer Institute SEER*Stat software. Version 8.2.1. https://seer.cancer.gov/seerstat/.

14. Surveillance, Epidemiology, and End Results (SEER) Program: SEER*Stat Database: Incidence - SEER 18 Regs Research Data + Hurricane Katrina Impacted Louisiana Cases, Nov 2014 Sub (2000-2012) <Katrina/Rita Population Adjustment> - Linked To County Attributes - Total U.S. 1969-2013 Counties, National Cancer Institute, DCCPS, Surveillance Research Program, Surveillance Systems Branch, released April 2015, based on the November 2014 submission. https://www.seer.cancer.gov. Accessed 3 Dec 2015.

15. Bell FC, Miller ML. Life Tables for the United States Social Security Area 1900-2100, Actuarial Study No. 120. https://www.ssa.gov/oact/NOTES/ as120/LifeTables_Tbl 6 2000.html. Accessed 27 Nov 2015.

16. Surveillance, Epidemiology, and End Results (SEER) Program: SEER*Stat Database: Incidence - SEER 9 Regs Research Data, Nov 2014 Sub (1973-2012) < Katrina/Rita Population Adjustment> - Linked To County Attributes - Total U.S., 1969-2013 Counties, National Cancer Institute, DCCPS, Surveillance Research Program, Surveillance Systems Branch, released April 2015, based on the November 2014 submission. https:// www.seer.cancer.gov. Accessed 3 Dec 2015.

17. Surveillance, Epidemiology, and End Results (SEER) Program: SEER*Stat Database: Incidence-Based Mortality - SEER 9 Regs Research Data, Nov 2014 Sub (1973-2012) < Katrina/Rita Population Adjustment> - Linked To County Attributes - Total U.S., 1969-2013 Counties, National Cancer Institute, DCCPS, Surveillance Research Program, Surveillance Systems Branch, released April 2015, based on the November 2014 submission. https://www.seer.cancer.gov. Accessed 25 Nov 2015

18. Nelson Q, Agarwal N, Stephenson R, Cannon-Albright LA. A population-based analysis of clustering identifies a strong genetic contribution to lethal prostate cancer. Front Genet. 2013. https://doi.org/ 10.3389/fgene.2013.00152.

19. Minikel EV, Zerr I, Collins SJ, Ponto C, Boyd A, Klug G, Karch A, Kenny J, Collinge J, Takada LT, Forner S, Fong JC, Mead S, Geschwind MD. Ascertainment bias causes false signal of anticipation in genetic prion disease. Am J Hum Genet. 2014. https://doi.org/10.1016/j.ajhg.2014.09.003.

20. Nussbaum RL, Mclnnes RR, Willard HF, Hamosh A. Patterns of single-gene inheritance. In: Thompson \& Thompson Genetics in Medicine. 7th edn. Philadelphia: Saunders/Elsevier; 2007. p. 115-49.

21. Boonstra PS, Gruber SB, Raymond VM, Huang SC, Timshel S, Nilbert M, Mukherjee B. A review of statistical methods for testing genetic anticipation: looking for an answer in lynch syndrome. Genet Epidemiol. 2010. https://doi.org/10.1002/gepi.20534. 